\title{
All-Multiplicity Nonplanar Amplitude Integrands in Maximally Supersymmetric Yang- Mills Theory at Two Loops
}

\author{
Jacob L. Bourjaily $\odot,{ }^{1,2,3}$ Enrico Herrmann $\odot,{ }^{4}$ Cameron Langer $\odot,{ }^{5}$ Andrew J. McLeod, ${ }^{1}$ and Jaroslav Trnka ${ }^{5}$ \\ ${ }^{1}$ Niels Bohr International Academy and Discovery Center, Niels Bohr Institute, University of Copenhagen, \\ Blegdamsvej 17, DK-2100 Copenhagen $\varnothing$, Denmark \\ ${ }^{2}$ Center for the Fundamental Laws of Nature, Department of Physics, Jefferson Physical Laboratory, \\ Harvard University, Cambridge, Massachusetts 02138, USA \\ ${ }^{3}$ Institute for Gravitation and the Cosmos, Department of Physics, Pennsylvania State University, \\ University Park, Pennsylvania 16892, USA \\ ${ }^{4}$ SLAC National Accelerator Laboratory, Stanford University, Stanford, California 94039, USA \\ ${ }^{5}$ Center for Quantum Mathematics and Physics (QMAP), Department of Physics, \\ University of California, Davis, California 95616, USA
}

(Received 23 December 2019; accepted 28 February 2020; published 20 March 2020)

We give a prescriptive representation of all-multiplicity two-loop maximally-helicity-violating (MHV) amplitude integrands in fully-color-dressed (nonplanar) maximally supersymmetric Yang-Mills theory.

DOI: 10.1103/PhysRevLett.124.111603

Introduction.-Recent years have been witness to tremendous advances in our understanding of-and our ability to compute-scattering amplitudes in perturbative quantum field theory (see, e.g., Refs. [1-6], and references therein). Perhaps the most impressive testament to these advances is found in the planar limit of maximally supersymmetric $(\mathcal{N}=4)$ Yang-Mills theory $(\mathrm{SYM})[7,8]$. In this theory, loop integrands can be recursed to all orders [9], with local formulas known at all particle multiplicities through three loops [10-12] and for four particles through ten loops [13-15]. Integrated expressions are also known for six particles through seven loops [16,17], and symbols are known for seven particles through four loops [18,19]. These computational triumphs only scratch the surface of the theoretical advances that have accompanied them (see, for example, Refs. [20-25]); having access to this increasingly substantial compendium of concrete "data" has unquestionably fueled more general progress.

In nonplanar theories, considerably less data are available. This is true even for the simplest quantum field theories, such as color-dressed (or "nonplanar") SYM and maximal $(\mathcal{N}=8)$ supergravity (SUGRA). In both theories, amplitude integrands are known for four particles through five loops [26-31], and for five or six particles only through a modest two loops [32-34]. (Notably, the three-loop fourparticle [35] and two-loop five-particle amplitudes have also recently been integrated [36-40].) It is known that the

Published by the American Physical Society under the terms of the Creative Commons Attribution 4.0 International license. Further distribution of this work must maintain attribution to the author(s) and the published article's title, journal citation, and DOI. Funded by SCOAP ${ }^{3}$. integrands for SUGRA amplitudes will involve terms with arbitrarily bad ultraviolet behavior (such as double poles at infinity) starting from seven particles [41]. In contrast, amplitude integrands in SYM are expected to be free of poles at infinity to all loop orders $[33,42,43]$. Therefore, these amplitude integrands should be expressible in terms of an integrand basis with "triangle power counting" (a notion whose precise definition beyond the planar limit will be described in a forthcoming work [44]).

In this Letter, we show that this is indeed the case by presenting the first fully explicit, color-dressed, prescriptive representation of all-multiplicity maximally-helicity-violating (MHV) amplitude integrands in SYM at two loops. In the spirit of the original "guess" at tree-level of Parke and Taylor [45] and similar guesses-later proven-at one $[46,47]$, two $[9,48]$, and three loops $[12,49]$ in the planar sector, we have checked that our result smoothly reproduces known results for 4 through 6 particles, and passes many nontrivial tests at higher multiplicity.

All-multiplicity MHV amplitude integrands.-The twoloop MHV all-multiplicity SYM integrand representation we construct in this work is explicitly prescriptive [12]: expressed in terms of a basis of integrands diagonalized with respect to a spanning set of field theory cuts (see, e.g., Refs. [50-54] for related work). Our basis consists of all two-loop integrands that have at most single poles at infinity. In terms of these, all integrands with more than $4 L$ propagators at $L$ loops are reducible, making the system explicitly triangular in cuts (and hence easy to diagonalize). It is worth pointing out that our setup leads to a surprisingly small number of relevant integrand basis elements in comparison to (for arbitrary $n$ ) the infinite number of Feynman diagrams required in traditional field theory 
methods (or even for BCJ [55], for example). Because individual integrands have support on poles at infinite loop momentum, the cancellation of these residues at infinity for SYM amplitudes [41] amounts to a nontrivial consistency check.

As our basis is diagonal in a spanning set of cuts, each integrand's coefficient is simply a residue of field theoryin our case, always a leading singularity (or zero). Thus, our representation takes the simple form:

$$
\mathcal{A}_{n}^{\mathrm{MHV},(2-\text { loop })}=\sum_{\substack{\text { (inequivalent) } \\ \text { leading singularticis } \mathfrak{f}}} \mathfrak{f} \times \mathcal{I}_{\mathfrak{f}} \text {, }
$$

where $\mathfrak{f}$ belongs to one of the six classes of (color-dressed) field theory leading singularities with MHV-helicity support given in Table I. The sum is over all distributions of external legs. Two leading singularities are considered equivalent if they are isomorphic as helicity-decorated graphs. Helicity degrees for $\mathrm{MHV} / \overline{\mathrm{MHV}}$ are indicated in Table I by blue and white vertices, respectively.

The representation Eq. (1) is a sum over all distinct leg distributions-including cases where the sets of legs $A, B$, $C$ attached to MHV vertices are allowed to be empty. Such leading singularities have the interpretation of a residue taken in a soft (and sometimes collinear) region, which sets the momentum flowing through the "doubled" propagator to zero. The numerators of integrands corresponding to such cases always become proportional to the doubled propagator, leaving us with an ordinary collection of Feynman propagators. For example,

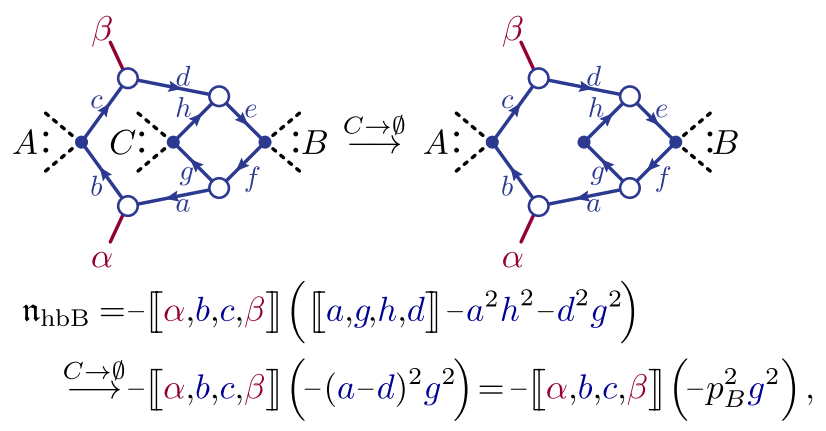

where $p_{B}^{2}:=\left(\sum_{b \in B} p_{b}\right)^{2}$. [Moreover, we can see that this numerator will vanish when the total momentum $B$ is massless (or empty).]

Note that our instruction to sum over "all" distributions of legs for the figures in Table I seems to include cases with massless triangles or even bubbles (as for hexabox B when $B=C=\varnothing)$; in all such cases, the corresponding integrand numerators either vanish or the contributions cancel in sum.

The leading singularities in Table I, appearing as coefficients $\mathfrak{f}$ in Eq. (1), should be understood as fullycolor-dressed on-shell functions in SYM. As such, every (tree-amplitude) vertex is fully (Bose) symmetric. Without any reference to a particular gauge group (or trace decomposition), these factors can be defined concretely in terms of locally (cyclically) ordered MHV on-shell diagrams $[56,57]$ and graphs built out of (graphs of) structure constants of the type considered in Ref. [58] (see also Refs. [59,60]). Explicit expressions for all these leading singularities were given in Appendix B of Ref. [34]. (These formulas are all smooth under taking any of the leg ranges $A, B, C$ to be empty, requiring no special cases in their definitions.)

Explicit integrand topologies and numerators.Attached to each leading singularity of Table I, we must construct an integrand that has unit support on the corresponding point in loop momentum space. Recall that when a MHV vertex in a leading singularity has no external legs attached to it, the corresponding residue is to be understood as the double constraint taking the momentum through that edge to be on shell and soft. Normalizing these integrands on their associated kinematic points is a good start, but is not sufficient to define our basis.

As stated above, our starting point is a complete basis of integrands (in four dimensions) with at most single poles at infinite loop momentum. In this space, there will be many integrands (numerator degrees of freedom) that can be normalized at points where no amplitudes in SYM have support. For example, integrands of the type shown in Table II are defined to have unit residue on a contour defined by cutting all seven propagators and symmetrically sending each loop to infinity. (When one or both of the leg ranges $A, B$ are empty, the numerator in Table II cancels the doubled propagator; the contour is then defined by starting

TABLE I. The six topology groups of two-loop leading singularities of MHV amplitudes. Explicit formulas are given in Ref. [34].

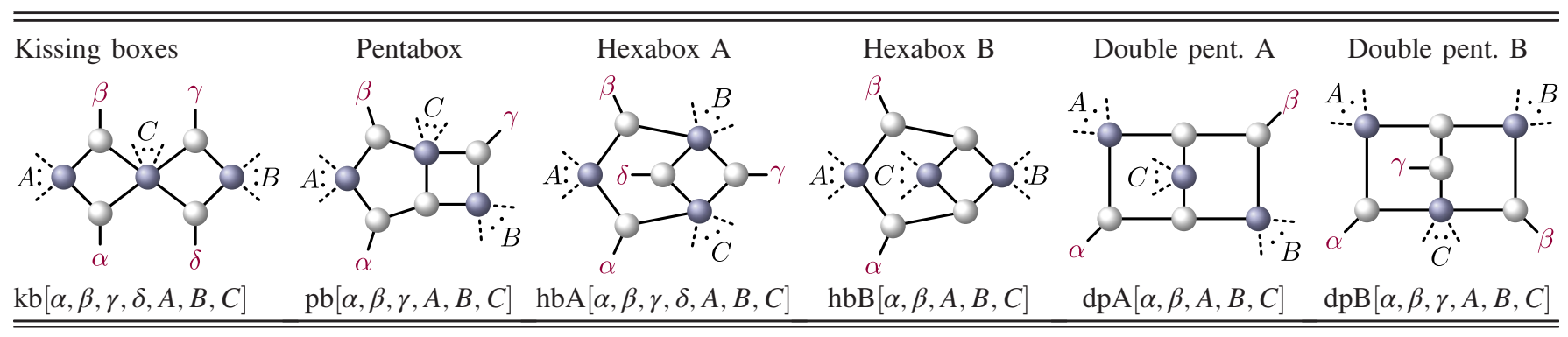


TABLE II. Basis integrands normalized at infinite loop momentum. In SYM, all these integrands have vanishing coefficients; nevertheless, all other integrands must be diagonalized with respect to these, accounting for the "contact terms" in the definition of integrands appearing in Table IV. (Integrands in Table III are automatically diagonal with respect to these.)

"contact terms" (normalized at $\infty$ )

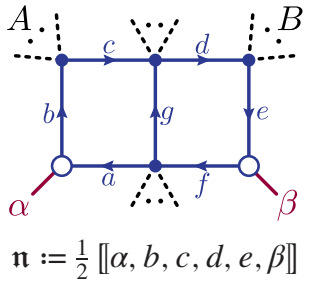

from the "heptacut" that takes all propagators on shell and for which the momentum flowing through any empty vertices is taken to be collinear to either $\alpha$ or $\beta$.) As all amplitude integrands in SYM should vanish at these points, the coefficients of these integrands in the sum (1) must be zero. Nevertheless, the entire (initially triangular) system of integrands must be diagonalized.

Besides points where general SYM amplitude integrands vanish, we take advantage of the particular simplicity of MHV amplitudes. Focusing on the simplest helicity configuration allows us to eliminate further integrand degrees of freedom by normalizing them on residues where MHV integrands have to vanish due to helicity selection rules. Note, however, that this procedure does not eliminate all integrand topologies without MHV support (see discussion below).

It turns out that the "leading" (noncontact) to be clear, we define contact terms as factors in the numerator proportional to one or more inverse propagators of the graph terms of the integrand numerators for six-particle amplitudes in Ref. [34] are automatically diagonal with respect to themselves. However, the naive numerators for two classes of integrands - the pentaboxes and double pentagons of type A-have support on the cuts defining the integrands of Table II. As such, diagonalization with respect to these "contact terms" results in some changes with respect to the naive numerators. Taking into account these minor rotations in the basis, we obtain the form of our answer. (It is worth pointing out that while the numerator of hexabox $\mathrm{B}$ in Table III appears to have contact terms, these should not be viewed as contact terms: they are fully fixed by graph symmetries, power counting, integral purity, and chirality.)

The resulting integrand basis we find is summarized in Tables III and IV. In Table III we list all the numerators, which are defined irrespective of whether or not any of the leg ranges $A, B, C$ are empty; in Table IV, we give expressions for the numerators of the pentabox and double pentagon A integrands, which require contact terms depending on whether the leg ranges $A$ and/or $B$ are empty.

These numerators are expressed in the notation

$$
\llbracket a_{1}, a_{2}, \ldots, c_{1}, c_{2} \rrbracket:=\left[\left(a_{1} \cdot a_{2}\right)_{\beta}^{\alpha} \cdots\left(c_{1} \cdot c_{2}\right)_{\alpha}^{\gamma}\right],
$$

where $\left(a_{1} \cdot a_{2}\right)_{\beta}^{\alpha}:=a_{1}^{\alpha \dot{\alpha}} \epsilon_{\dot{\alpha} \dot{\gamma}} a_{2}^{\dot{\gamma} \gamma} \epsilon_{\gamma \beta}$ and $a^{\alpha \dot{\alpha}}=a^{\mu} \sigma_{\mu}^{\alpha \dot{\alpha}}$ are " $2 \times 2$ " four-momenta, defined via the Pauli matrices. (Our $\llbracket \cdots \rrbracket$ may be more familiar if written as $\operatorname{tr}_{+}[\cdots]$.)

Cancellation of Calabi-Yau cut components.-As explained in Ref. [34], local integrand representations of MHV amplitudes require terms that individually have support on elliptic and $K 3$ (Calabi-Yau) subtopologies [61-64]. This is despite the fact that these amplitudes are unquestionably polylogarithmic. The easiest way to see this is to note that after cutting the six propagators of the "tardigrade" integral [63]

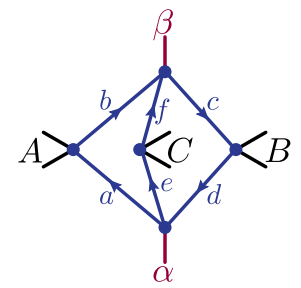

there is no helicity flow consistent with MHV. The same argument applies when any one of $A, B, C$ becomes massless - the case first relevant to seven particles, where

TABLE III. Integrand topologies with numerators that are smooth under all degenerations to empty leg-ranges.

\begin{tabular}{|c|c|c|c|}
\hline Kissing boxes & Hexabox A & Hexabox B & Double pentagon B \\
\hline $\begin{array}{ll}1 & \\
\alpha & \delta\end{array}$ & $\therefore \dot{C}$ & F & 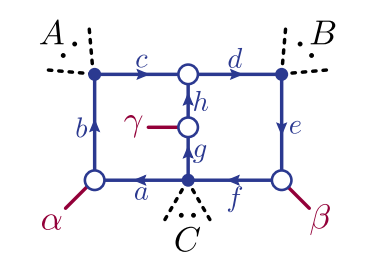 \\
\hline $\begin{array}{l}\mathcal{I}_{\mathrm{kb}}[\alpha, \beta, \gamma, \delta, A, B, C] \\
\mathfrak{n}:=\llbracket \alpha, b, c, \beta \rrbracket \llbracket \gamma, f, g, \delta \rrbracket\end{array}$ & $\begin{array}{c}\mathcal{I}_{\mathrm{hbA}}[\alpha, \beta, \gamma, \delta, A, B, C] \\
\mathfrak{n}:=\llbracket \alpha, b, c, \beta \rrbracket \llbracket \gamma, f, g, \delta \rrbracket\end{array}$ & $\begin{array}{c}\mathcal{I}_{\mathrm{hbB}}[\alpha, \beta, A, B, C] \\
\mathfrak{n}:=-\llbracket \alpha, b, c, \beta \rrbracket(\llbracket a, g, h, d \rrbracket \\
\left.-a^{2} h^{2}-d^{2} g^{2}\right)\end{array}$ & $\begin{aligned} & \mathcal{I}_{\mathrm{dpB}}[\alpha, \beta, \gamma, A, B, C] \\
\mathfrak{n}:= & \frac{1}{2}(\llbracket \alpha, b, c, d, e, \beta, g, h \rrbracket \\
& -\llbracket \alpha, b, c, d, e, \beta, h, g \rrbracket)\end{aligned}$ \\
\hline
\end{tabular}


TABLE IV. Integrand topologies with numerators that have contact terms that change when some leg ranges are empty.

$$
\begin{aligned}
& \text { Pentabox } \\
& A \because \because \gamma_{\alpha}^{c} \\
& \mathcal{I}_{\mathrm{pb}}[\alpha, \beta, \gamma, A, B, C] \\
& \mathfrak{n}:=-\llbracket \alpha, b, c, \beta \rrbracket \llbracket \gamma, f, g, a \rrbracket \\
& -\frac{1}{2} \llbracket \alpha, b, c, \beta, \gamma, f, g, a \rrbracket \\
& +\mathfrak{n}_{\mathrm{pb}}^{c} \\
& \text { where } \\
& \mathfrak{n}_{\mathrm{pb}}^{c}:=\frac{1}{2} \begin{cases}0 & \text { if } A \neq \varnothing, B \neq \varnothing \\
b^{2} d^{2} \llbracket \gamma, f, g, \alpha \rrbracket & \text { if } A \neq \varnothing, B \neq \varnothing \\
f^{2} d^{2} \llbracket \alpha, b, c, \gamma \rrbracket-f^{2} c^{2} \llbracket \alpha, b, d, \gamma \rrbracket & \text { if } B \neq \varnothing\end{cases} \\
& \mathfrak{n}_{\mathrm{dpA}}^{c}:=\frac{1}{2} \begin{cases}0 & \text { if } A \neq \varnothing, B \neq \varnothing \\
b^{2}\left(g^{2}+h^{2}\right) \llbracket \beta, e, f, \alpha \rrbracket & \text { if } A \neq \varnothing, B \neq \varnothing \\
e^{2}\left(g^{2}+h^{2}\right) \llbracket \alpha, b, c, \beta \rrbracket & \text { if } B \neq \varnothing\end{cases} \\
& \mathfrak{n}:=-\llbracket \alpha, b, c, h, g, f, e, \beta \rrbracket \\
& +\mathfrak{n}_{\mathrm{dpA}}^{c}
\end{aligned}
$$

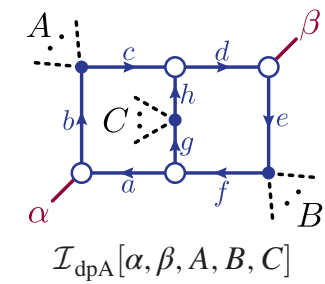

$$
\begin{aligned}
& \left.\frac{\llbracket \alpha, a, b, \beta \rrbracket\left(\llbracket a-\alpha, e, f, b+\beta \rrbracket-(a-\alpha)^{2} f^{2}-(b+\beta)^{2} e^{2}\right)}{(a-\alpha)^{2}(b+\beta)^{2}}\right|_{\text {cut }} \\
& =-\left.\frac{\llbracket \alpha, a, b, f, e, d, c, \beta \rrbracket}{(a-\alpha)^{2}(c-\beta)^{2}}\right|_{\mathrm{cut}}=-\left.\frac{\llbracket \alpha, a, b, c, d, e, f, \beta \rrbracket}{(a-\alpha)^{2}(f-\beta)^{2}}\right|_{\mathrm{cut}},
\end{aligned}
$$

where the first line is minus the integrand for the hbA contribution. To make better sense of Eq. (7), notice that we have labeled the integrands (5) decorating the leading singularities of Eq. (6) according to the cut specified in Eq. (4) and evaluated the numerators defined in Tables III and IV in terms of these loop-momentum labels.

Further consistency checks.-Besides the cancellation of the Calabi-Yau hexacut, we have performed a number of nontrivial consistency checks of our new result. In particular, we have explicitly compared the two-loop four-, five-, and six-particle integrands to known results $[26,32,34]$. Furthermore, in the planar sector we compared our result to that obtained from loop-recursion relations [9]. We did not check all eight-particle unitarity cuts; however, we made sure that our answer passes a large number of fiveand six-cut checks, which involve almost all integrand topologies of our answer. (No new integrand topologies appear beyond eight particles.) Matching these low cuts correctly constitutes a highly nontrivial consistency check on our result.

Infrared divergences and infrared finiteness.-The structure of infrared divergences has played a major role in understanding fundamental properties of gauge theories; see, e.g., Refs. [66-73]. As with the representation found for six particles in Ref. [34], the representation of MHV amplitudes in Eq. (1) manifests as much of the infrared structure of the theory as possible. Specifically, all of the soft and collinear regions of loop-momentum space related to infrared divergences of amplitudes are matched manifestly, with coefficients that directly suggest something like exponentiation. By this, we refer to the fact that these leading singularities directly connect to lower-loop integrands (or trees) times products of factors that manifestly encode one-loop divergences. The precise sense in which this has something to say about how infrared divergences are organized in this representation-as compared with Ref. [67], for example-remains to be explored. 
One consequence of matching these infrared-divergent leading singularities directly (and diagonalizing our basis with respect to them) is that a large fraction of the terms in our basis are infrared finite. In particular, only about half of the integrals required for six particles are infrared divergent, and this fraction gets smaller at higher multiplicity. We strongly suspect that this feature will prove helpful in eventually finding analytic expressions for these amplitudes once regulated in some particular scheme.

It is worth emphasizing that the integrand we have constructed is strictly four dimensional, and therefore not immediately suitable for dimensional regularization: in 4-2 $\epsilon$ dimensions, the integrand coefficients would change by terms of $\mathcal{O}(\epsilon)$, some of which survive as $\epsilon \rightarrow 0$. (It would be interesting to know dimensionally regularized integrands for these amplitudes, but constructing them would go beyond the scope of our present work.) That being said, the infrared structure of these four-dimensional integrands can be studied without subtlety using a Higgs or mass regulator [74,75], as any $\mathcal{O}\left(m^{2}\right) \log \left(m^{2}\right)$ terms would vanish in the $m^{2} \rightarrow 0$ limit.

Conclusions and discussion.-At tree level and one loop, all amplitudes are (built from terms that are) planar with respect to some ordering. In this Letter we have given the first all-multiplicity formula for genuinely nonplanar scattering amplitude integrands. Our strategy avoided any reference to any particular gauge group, and required no choice of loop-momentum labels or routing. As such, we have demonstrated the power of prescriptive unitarity beyond the planar limit, opening the door to many future applications, including a better understanding of the structure of perturbative quantum field theory.

In the Supplemental Material [76] we have prepared a Mathematica notebook making use of Eq. (1). Specifically, we provide documented functions to decompose, evaluate, and expand into color traces all leading singularities in Table I, represent and evaluate each of the integrands in Tables III and IV, and to generate permutation-class representatives of each term appearing in Eq. (1) (together with the entire permutation sums) for arbitrary multiplicity. Useful tools for working with amplitudes in Mathematica can be found in Refs. [77,78].

This work was supported in part by the Danish National Research Foundation (DNRF91), a grant from the Villum Fonden, a Starting Grant (No. 757978) from the European Research Council (J.L.B., A. J. M.), and a Carlsberg Postdoctoral Fellowship (CF18-0641) (A. J. M.). The research of J.T. and C.L. is supported in part by U.S. Department of Energy Grant No. DE-SC0009999 and by the funds of University of California. E. H. is supported by the U.S. Department of Energy under Contract No. DEAC02-76SF00515. Finally, J. L. B., E. H., and A. J. M. are grateful for the hospitality of the Aspen Center for Physics, which is supported by National Science Foundation Grant No. PHY-1607611, and also to the Harvard Center for Mathematical Sciences and Applications.

[1] M. L. Mangano and S. J. Parke, Multiparton amplitudes in gauge theories, Phys. Rep. 200, 301 (1991).

[2] L. J. Dixon, Calculating scattering amplitudes efficiently, arXiv:hep-ph/9601359.

[3] H. Elvang and Y.-t. Huang, Scattering amplitudes, arXiv: 1308.1697.

[4] J. M. Henn and J. C. Plefka, Scattering amplitudes in gauge theories, Lect. Notes Phys. 883, 1 (2014).

[5] L. J. Dixon, A brief introduction to modern amplitude methods, in Proceedings of the 2014 Theoretical Advanced Study Institute in Elementary Particle Physics: Journeys Through the Precision Frontier: Amplitudes for Colliders (TASI 2014), Boulder, 2014 (2015), pp. 39-97.

[6] C. Cheung, TASI lectures on scattering amplitudes, in Proceedings of the 2016 Theoretical Advanced Study Institute in Elementary Particle Physics: Anticipating the Next Discoveries in Particle Physics (TASI 2016), Boulder, 2016 (2018), pp. 571-623.

[7] L. Brink, J. H. Schwarz, and J. Scherk, Supersymmetric Yang-Mills theories, Nucl. Phys. B121, 77 (1977).

[8] F. Gliozzi, J. Scherk, and D. I. Olive, Supersymmetry, supergravity theories and the dual spinor model, Nucl. Phys. B122, 253 (1977).

[9] N. Arkani-Hamed, J. L. Bourjaily, F. Cachazo, S. CaronHuot, and J. Trnka, The all-loop integrand for scattering amplitudes in planar $\mathcal{N}=4$ SYM, J. High Energy Phys. 01 (2011) 041.

[10] J. L. Bourjaily, S. Caron-Huot, and J. Trnka, Dualconformal regularization of infrared loop divergences and the chiral box expansion, J. High Energy Phys. 01 (2015) 001.

[11] J. L. Bourjaily and J. Trnka, Local integrand representations of all two-loop amplitudes in planar SYM, J. High Energy Phys. 08 (2015) 119.

[12] J. L. Bourjaily, E. Herrmann, and J. Trnka, Prescriptive unitarity, J. High Energy Phys. 06 (2017) 059.

[13] J. L. Bourjaily, A. DiRe, A. Shaikh, M. Spradlin, and A. Volovich, The soft-collinear bootstrap: $\mathcal{N}=4$ Yang-Mills amplitudes at six and seven loops, J. High Energy Phys. 03 (2012) 032.

[14] J. L. Bourjaily, P. Heslop, and V.-V. Tran, Perturbation Theory at Eight Loops: Novel Structures and the Breakdown of Manifest Conformality in $\mathcal{N}=4$ Supersymmetric Yang-Mills Theory, Phys. Rev. Lett. 116, 191602 (2016).

[15] J. L. Bourjaily, P. Heslop, and V.-V. Tran, Amplitudes and correlators to ten loops using simple, graphical bootstraps, J. High Energy Phys. 11 (2016) 125.

[16] S. Caron-Huot, L. J. Dixon, F. Dulat, M. von Hippel, A. J. McLeod, and G. Papathanasiou, Six-gluon amplitudes in planar $\mathcal{N}=4$ super-Yang-Mills theory at six and seven loops, J. High Energy Phys. 08 (2019) 016.

[17] S. Caron-Huot, L. J. Dixon, F. Dulat, M. von Hippel, A. J. McLeod, and G. Papathanasiou, The cosmic Galois Group 
and extended Steinmann relations for planar $\mathcal{N}=4 \mathrm{SYM}$ amplitudes, J. High Energy Phys. 09 (2019) 061.

[18] L. J. Dixon, J. Drummond, T. Harrington, A. J. McLeod, G. Papathanasiou, and M. Spradlin, Heptagons from the Steinmann cluster bootstrap, J. High Energy Phys. 02 (2017) 137.

[19] J. Drummond, J. Foster, Ö. Gürdoğan, and G. Papathanasiou, Cluster adjacency and the four-loop NMHV heptagon, J. High Energy Phys. 03 (2019) 087.

[20] J. Drummond, J. Henn, V. Smirnov, and E. Sokatchev, Magic identities for conformal four-point integrals, J. High Energy Phys. 01 (2007) 064.

[21] N. Beisert, C. Ahn, L. F. Alday, Z. Bajnok, J. M. Drummond et al., Review of AdS/CFT integrability: An overview, Lett. Math. Phys. 99, 3 (2012).

[22] N. Arkani-Hamed, J. Bourjaily, F. Cachazo, and J. Trnka, Unification of residues and Grassmannian dualities, J. High Energy Phys. 01 (2011) 049.

[23] N. Arkani-Hamed, J. Bourjaily, F. Cachazo, and J. Trnka, Local spacetime physics from the Grassmannian, J. High Energy Phys. 01 (2011) 108.

[24] N. Arkani-Hamed, J. L. Bourjaily, F. Cachazo, A. B. Goncharov, A. Postnikov, and J. Trnka, Scattering amplitudes and the positive Grassmannian, arXiv:1212.5605.

[25] N. Arkani-Hamed and J. Trnka, The amplituhedron, J. High Energy Phys. 10 (2014) 30.

[26] Z. Bern, J. Rozowsky, and B. Yan, Two-loop four-gluon amplitudes in $\mathcal{N}=4$ SuperYang-Mills, Phys. Lett. B 401, 273 (1997).

[27] Z. Bern, L. J. Dixon, D. C. Dunbar, M. Perelstein, and J. S. Rozowsky, On the relationship between Yang-Mills theory and gravity and its implication for ultraviolet divergences, Nucl. Phys. B530, 401 (1998).

[28] Z. Bern, J. J. M. Carrasco, L. J. Dixon, H. Johansson, D. A. Kosower, and R. Roiban, Three-Loop Superfiniteness of $\mathcal{N}=8$ Supergravity, Phys. Rev. Lett. 98, 161303 (2007).

[29] Z. Bern, J. J. M. Carrasco, L. J. Dixon, H. Johansson, and R. Roiban, The complete four-loop four-point amplitude in $\mathcal{N}=4$ super-Yang-Mills theory, Phys. Rev. D 82, 125040 (2010).

[30] Z. Bern, J. J. M. Carrasco, H. Johansson, and R. Roiban, The Five-Loop Four-Point Amplitude of $\mathcal{N}=4$ SuperYang-Mills Theory, Phys. Rev. Lett. 109, 241602 (2012).

[31] Z. Bern, J. J. M. Carrasco, W.-M. Chen, H. Johansson, R. Roiban, and M. Zeng, The five-loop four-point integrand of $\mathcal{N}=8$ supergravity as a generalized double copy, Phys. Rev. D 96, 126012 (2017).

[32] J. J. M. Carrasco and H. Johansson, Five-point amplitudes in $\mathcal{N}=4$ super-Yang-Mills theory and $\mathcal{N}=8$ supergravity, Phys. Rev. D 85, 025006 (2012).

[33] Z. Bern, E. Herrmann, S. Litsey, J. Stankowicz, and J. Trnka, Evidence for a nonplanar amplituhedron, J. High Energy Phys. 06 (2016) 098.

[34] J. L. Bourjaily, E. Herrmann, C. Langer, A. J. McLeod, and J. Trnka, Prescriptive unitarity for non-planar six-particle amplitudes at two loops, J. High Energy Phys. 12 (2019) 073.

[35] J. M. Henn and B. Mistlberger, Four-Gluon Scattering at Three Loops, Infrared Structure, and the Regge Limit, Phys. Rev. Lett. 117, 171601 (2016).
[36] S. Abreu, L. J. Dixon, E. Herrmann, B. Page, and M. Zeng, The Two-Loop Five-Point Amplitude in $\mathcal{N}=4$ SuperYang-Mills Theory, Phys. Rev. Lett. 122, 121603 (2019).

[37] D. Chicherin, T. Gehrmann, J. M. Henn, P. Wasser, Y. Zhang, and S. Zoia, All Master Integrals for Three-Jet Production at Next-to-Next-to-Leading Order, Phys. Rev. Lett. 123, 041603 (2019).

[38] D. Chicherin, T. Gehrmann, J. M. Henn, P. Wasser, Y. Zhang, and S. Zoia, Analytic Result for a Two-Loop FiveParticle Amplitude, Phys. Rev. Lett. 122, 121602 (2019).

[39] D. Chicherin, T. Gehrmann, J. M. Henn, P. Wasser, Y. Zhang, and S. Zoia, The two-loop five-particle amplitude in $\mathcal{N}=8$ supergravity, J. High Energy Phys. 03 (2019) 115.

[40] S. Abreu, L. J. Dixon, E. Herrmann, B. Page, and M. Zeng, The two-loop five-point amplitude in $\mathcal{N}=8$ supergravity, J. High Energy Phys. 03 (2019) 123.

[41] J. L. Bourjaily, E. Herrmann, and J. Trnka, Amplitudes at infinity, Phys. Rev. D 99, 066006 (2019).

[42] N. Arkani-Hamed, J. L. Bourjaily, F. Cachazo, and J. Trnka, Singularity Structure of Maximally Supersymmetric Scattering Amplitudes, Phys. Rev. Lett. 113, 261603 (2014).

[43] Z. Bern, E. Herrmann, S. Litsey, J. Stankowicz, and J. Trnka, Logarithmic singularities and maximally supersymmetric amplitudes, J. High Energy Phys. 06 (2015) 202.

[44] J. L. Bourjaily, E. Herrmann, and J. Trnka, Building bases of loop integrands (to be published).

[45] S. J. Parke and T. R. Taylor, An Amplitude for $n$-Gluon Scattering, Phys. Rev. Lett. 56, 2459 (1986).

[46] Z. Bern, G. Chalmers, L. J. Dixon, and D. A. Kosower, OneLoop $N$ Gluon Amplitudes with Maximal Helicity Violation via Collinear Limits, Phys. Rev. Lett. 72, 2134 (1994).

[47] Z. Bern, L. J. Dixon, D. C. Dunbar, and D. A. Kosower, One-loop $n$-point gauge theory amplitudes, unitarity and collinear limits, Nucl. Phys. B425, 217 (1994).

[48] C. Vergu, The two-loop MHV amplitudes in $\mathcal{N}=4$ supersymmetric Yang-Mills theory, Phys. Rev. D 80, 125025 (2009).

[49] N. Arkani-Hamed, J. L. Bourjaily, F. Cachazo, and J. Trnka, Local integrals for planar scattering amplitudes, J. High Energy Phys. 06 (2012) 125.

[50] B. Feng and R. Huang, The classification of two-loop integrand basis in pure four-dimension, J. High Energy Phys. 02 (2013) 117.

[51] G. Ossola, C. G. Papadopoulos, and R. Pittau, Reducing full one-loop amplitudes to scalar integrals at the integrand level, Nucl. Phys. B763, 147 (2007).

[52] P. Mastrolia, E. Mirabella, G. Ossola, and T. Peraro, Integrand-reduction for two-loop scattering amplitudes through multivariate polynomial division, Phys. Rev. D 87, 085026 (2013).

[53] P. Mastrolia, E. Mirabella, G. Ossola, and T. Peraro, Multiloop integrand reduction for dimensionally regulated amplitudes, Phys. Lett. B 727, 532 (2013).

[54] S. Abreu, F. Febres Cordero, H. Ita, M. Jaquier, B. Page, and M. Zeng, Two-Loop Four-Gluon Amplitudes with the Numerical Unitarity Method, Phys. Rev. Lett. 119, 142001 (2017).

[55] Z. Bern, J. J. M. Carrasco, and H. Johansson, New relations for gauge-theory amplitudes, Phys. Rev. D 78, 085011 (2008). 
[56] N. Arkani-Hamed, J. L. Bourjaily, F. Cachazo, A. Postnikov, and J. Trnka, On-shell structures of MHV amplitudes beyond the planar limit, J. High Energy Phys. 06 (2015) 179.

[57] J. L. Bourjaily, S. Franco, D. Galloni, and C. Wen, Stratifying on-shell cluster varieties: The geometry of non-planar on-shell diagrams, J. High Energy Phys. 10 (2016) 003.

[58] V. Del Duca, L. J. Dixon, and F. Maltoni, New color decompositions for gauge amplitudes at tree and loop level, Nucl. Phys. B571, 51 (2000).

[59] A. Ochirov and B. Page, Full colour for loop amplitudes in Yang-Mills theory, J. High Energy Phys. 02 (2017) 100.

[60] A. Ochirov and B. Page, Multi-quark colour decompositions from unitarity, J. High Energy Phys. 10 (2019) 058.

[61] J. L. Bourjaily, A. J. McLeod, M. Spradlin, M. von Hippel, and M. Wilhelm, Elliptic Double-Box Integrals: Massless Scattering Amplitudes beyond Polylogarithms, Phys. Rev. Lett. 120, 121603 (2018).

[62] J. L. Bourjaily, Y.-H. He, A. J. McLeod, M. von Hippel, and M. Wilhelm, Traintracks through Calabi-Yaus: Amplitudes beyond Elliptic Polylogarithms, Phys. Rev. Lett. 121, 071603 (2018).

[63] J. L. Bourjaily, A. J. McLeod, M. von Hippel, and M. Wilhelm, A (Bounded) Bestiary of Feynman Integral Calabi-Yau Geometries, Phys. Rev. Lett. 122, 031601 (2019).

[64] J. L. Bourjaily, A. J. McLeod, C. Vergu, M. Volk, M. von Hippel, and M. Wilhelm, Embedding Feynman integral (Calabi-Yau) geometries in weighted projective space, J. High Energy Phys. 01 (2020) 078.

[65] E. Herrmann and J. Trnka, Gravity on-shell diagrams, J. High Energy Phys. 11 (2016) 136.

[66] S. Weinberg, Infrared photons and gravitons, Phys. Rev. 140, B516 (1965).
[67] S. Catani, The singular behavior of QCD amplitudes at two loop order, Phys. Lett. B 427, 161 (1998).

[68] Z. Bern, V. Del Duca, and C. R. Schmidt, The infrared behavior of one loop gluon amplitudes at next-to-next-toleading order, Phys. Lett. B 445, 168 (1998).

[69] S. M. Aybat, L. J. Dixon, and G. F. Sterman, The two-loop soft anomalous dimension matrix and resummation at nextto-next-to leading pole, Phys. Rev. D 74, 074004 (2006).

[70] L. J. Dixon, L. Magnea, and G. F. Sterman, Universal structure of subleading infrared poles in gauge theory amplitudes, J. High Energy Phys. 08 (2008) 022.

[71] E. Gardi and L. Magnea, Factorization constraints for soft anomalous dimensions in QCD scattering amplitudes, J. High Energy Phys. 03 (2009) 079.

[72] T. Becher and M. Neubert, Infrared Singularities of Scattering Amplitudes in Perturbative QCD, Phys. Rev. Lett. 102 (2009) 162001; Erratum, 111, 199905 (2013).

[73] I. Feige and M. D. Schwartz, Hard-soft-collinear factorization to all orders, Phys. Rev. D 90, 105020 (2014).

[74] L. F. Alday, J. M. Henn, J. Plefka, and T. Schuster, Scattering into the fifth dimension of $\mathcal{N}=4$ super Yang-Mills, J. High Energy Phys. 01 (2010) 077.

[75] J. M. Henn, S. G. Naculich, H. J. Schnitzer, and M. Spradlin, Higgs-regularized three-loop four-gluon amplitude in $\mathcal{N}=4$ SYM: Exponentiation and Regge limits, J. High Energy Phys. 04 (2010) 038.

[76] See Supplemental Material at http://link.aps.org/ supplemental/10.1103/PhysRevLett.124.111603 for Mathematica code that implements the integrands presented in this paper.

[77] J. L. Bourjaily, Efficient tree-amplitudes in $\mathcal{N}=4$ : Automatic BCFW recursion in Mathematica, arXiv:1011.2447.

[78] J. L. Bourjaily, Positroids, plabic graphs, and scattering amplitudes in Mathematica, arXiv:1212.6974. 